\title{
DRAFT: NONLINEAR HYDRODYNAMICS OF A BLUFF-BODY STABILIZED TURBULENT PREMIXED FLAME
}

\author{
C.Y. Lee* R.S. Cant \\ Department of Engineering \\ University of Cambridge \\ Cambridge, CB2 1PZ \\ Email: cyl36@cam.ac.uk
}

\begin{abstract}
Bluff-body stabilized turbulent premixed flames can experience hydrodynamic instability caused by the interaction of the flame with small-scale vortices in the separated shear layer around the recirculation region, as well as with the large-scale coherent structures in the far-wake. A globally hydrodynamically unstable system, for example one which involves vortex shedding, can exhibit limit-cycle behaviour due to the coupling between pressure oscillation and velocity fluctuations. In this work, the hydrodynamic behaviour of a bluff-body stabilized turbulent premixed propane/air flame in a model jet-engine afterburner is investigated using Computational Fluid Dynamics (CFD). A URANS approach was found to be appropriate for the range of frequencies considered in this study. Combustion is modelled using a modified flame surface density (FSD) approach. The observed self-excited hydrodynamic oscillations are analyzed using a nonlinear dynamical framework which is capable of capturing elaborate nonlinear behaviour including quasiperiodicity and chaos. The results from the CFD are first validated using available experimental data. The velocity at the inlet is gradually increased from $14 \mathrm{~m} / \mathrm{s}$ to $33 \mathrm{~m} / \mathrm{s}$ and the global flame structure is observed. With increasing inlet velocity, the flame first transitions from steady state to an oscillating state with a symmetrical flame structure, and eventually to an asymmetrical flame structure at higher velocities. The flame is essentially steady in the lower range of velocities considered before transitioning to a limit cycle oscillation after a critical velocity is exceeded.
\end{abstract}

\footnotetext{
* Address all correspondence to this author.
}

A doubling in the frequency of the hydrodynamic oscillation is also observed at intermediate values of inlet velocity. This investigation demonstrates that turbulent premixed reacting flows can exhibit strong hydrodynamic oscillation. An understanding of such behaviour can assist in developing methods to control flow instabilities and therefore help in suppressing thermoacoustic oscillation.

\section{NOMENCLATURE}

\section{Roman Symbols}

$a_{c} \quad$ Hydrodynamic strain at extinction

a Hydrodynamic strain

C Correlation sum

c Reaction progress variable

$D_{c} \quad$ Correlation dimension

$f \quad$ Frequency

$i \quad$ Hypersphere

$I_{O} \quad$ Strain correction factor

$K \quad$ Stiffness parameter

$k \quad$ Turbulent kinetic energy

$\hat{L}_{y} \quad$ Flame wrinkling length scale

$m$ Embedding dimension

Ma Markstein number

$p \quad$ Pressure

$p_{\infty} \quad$ Reference pressure

$R \quad$ Euclidean radius

$S_{L} \quad$ Unstrained laminar flame speed

$T \quad$ Temperature 


$\begin{array}{ll}t & \text { Time } \\ t_{r} & \text { Laminar flame residence time } \\ u & \text { Velocity } \\ u_{\text {inlet }} & \text { Inlet velocity } \\ u^{\prime} & \text { Turbulence intensity } \\ w_{f} & \text { Bluff-body width } \\ Y & \text { Mass fraction } \\ \text { Greek } & \text { Symbols } \\ \varepsilon & \text { Turbulent dissipation } \\ \mathscr{L} & \text { Characteristic of pressure wave } \\ v & \text { Kinematic viscosity } \\ \omega & \text { Chemical reaction rate } \\ \phi & \text { Equivalence ratio } \\ \rho & \text { Density } \\ \mathbb{R}^{m} & \text { Euclidean space } \\ \Sigma & \text { Flame surface area per unit volume } \\ \tau & \text { Time delay } \\ \text { Subscripts } \\ P & \text { Product } \\ R & \text { Reactant } \\ \text { Other } & \text { Symbols } \\ - & \text { Time averaged } \\ l & \text { Fluctuation about a Favre average } \\ l & \text { Fluctuation about a time average } \\ \sim & \text { Favre averaged } \\ \text { Acronyms } \\ B M L & \text { Bray-Moss-Libby } \\ B V K & \text { Bernard von-Karman } \\ C F D & \text { Computational Fluid Dynamics } \\ F S D & \text { Flame surface density } \\ \text { ODE } & \text { Ordinary differential equation } \\ O p e n F & \text { OAM Open Field Operation and Manipulation } \\ P I S O & \text { Pressure Implicit Splitting of Operators } \\ U R A N S & \text { Unsteady Reynolds Averaged Navier Stokes }\end{array}$

\section{INTRODUCTION}

Stabilization of turbulent premixed flames in fast flowing gases is important to a range of combustion systems, including gas turbines, industrial burners, ramjets and afterburners. One means to achieve a stable flame is by using a bluff-body to generate a recirculation zone [1]. In this region, recirculating burned gases at high temperature act as a source of continuous heating of unburned reactants in the free stream. However, a stable flame can only be achieved within a limited range of fuel/air equivalence ratio and approach flow velocity. Beyond these operating limits, the flame can experience various instabilities that can lead to global extinction [2,3]. For example, the flame can be subjected to strong hydrodynamic oscillations, primarily due to large-scale vortices that tend to perturb the flame front and affect the local heat release rate $[4,5]$. Another commonly ob- served phenomenon associated with flame instabilities is thermoacoustic oscillation, under which the pressure fluctuations and unsteady heat release are in-phase [6]. Thermoacoustic oscillation is highly undesirable as it can affect the operation and structural integrity of the combustion system.

A wake-stabilized turbulent premixed flame such as that found in a jet-engine afterburner configuration is highly susceptible to hydrodynamic instabilities [7]. Large-scale motions such as Karman type vortex shedding or shear-layer instabilities are often observed in such a configuration. These flow instabilities tend to be more prominent under isothermal conditions than in reacting flows, as the combustion process introduces additional physics including exothermicity and flow dilatation that are absent in non-reacting bluff-body flows, as evidenced in experiments $[8,9]$ and numerical simulations [10-12]. In the presence of a flame, thermal expansion acts as a sink for vorticity, while baroclinic torque tends to counteract fluid-mechanical straining effects $[1,13,14]$. Nevertheless, the interaction between the flow, including the large-scale coherent structures and the small-scale turbulent vortices and the flame can affect the global flame behaviour. Velocity fluctuations caused by large-scale flow structures can lead to changes in the flame surface area [15] and entrainment of fresh mixture into hot products in the wake of flameholders [16]. Moreover, the dynamics of the flame front are strongly influenced by the hydrodynamic straining and curvature [17]. Fine-scale turbulence can affect the heat release due to changes in the local flame structure. Hydrodynamic oscillations can also interact with pressure waves in a confined chamber. If a system exhibits long range feedback involving pressure-velocity coupling, a limit cycle would be expected and this will in turn affect in the manner in which the flame behaves.

In this work, the hydrodynamic behaviour of a bluff-body stabilized turbulent premixed propane/air flame in a model afterburner is investigated using Computational Fluid Dynamics (CFD). To simulate the bluff-body stabilized flame, an unsteady Reynolds-averaged Navier Stokes (URANS) approach using the modified flame surface density (FSD) combustion model $[18,19]$ has been adopted. As low frequency flow instabilities such as those associated with shedding of vortices past the bluff-body or rollup of the flame front are of interest, a URANS approach is adequate. The modified FSD approach can effectively capture changes in the flame surface area due to straining and curvature effects, which has proved to be useful in modelling the response of premixed flames interacting with large-scale coherent flow structures [5]. Previous work in this area has demonstrated that by using a well-resolved URANS approach, fully developed combustion oscillations can be captured [20,21]. Successful comparison against experimental data has been carried out $[19,22]$, and theoretical methods have been able to predict stability boundaries [23]. The current work builds upon the work of Armitage et al. [22] that employed the URANS approach to study flame instability in the presence of vortex shedding and 
rollup, and gain insight into the origins of nonlinear behaviour during combustion oscillations.

Previous analysis of combustion instabilities has been carried out in both the frequency domain and the time domain. Frequency domain analysis is usually based on the Flame Describing Function [24, 25], and allows for the prediction of frequencies and amplitudes associated with a particular mode of oscillation. In this work, a time-domain analysis is employed [26, 27]. The observed self-excited hydrodynamic oscillations are investigated using a nonlinear dynamical framework [28]. Such an approach does not implicitly assume that a system exhibits only a limit cycle, and instead is capable of capturing elaborate nonlinear behaviour including period-doubling, quasiperiodicity and chaos [27]. The present work aims to demonstrate the nonlinear behaviour of the flame with changes in the inlet velocity. The flow behaviour past the bluff-body and far-wake region is known to be sensitive to variations of the inlet velocity. This will have an implication for the global flame dynamics and the nonlinear behaviour of the system.

The first part of the work reported in this paper serves to validate the URANS results for the current configuration. In the subsequent section, the inlet velocity of the wake-stablilized flame is varied and six flames at different inlet velocities are simulated. For each flame, the self-excited hydrodynamic response is examined, revealing nonlinear behaviour and the corresponding bifurcations. Changes in the flame structure due to hydrodynamic instability and the dynamics of the system are elucidated using the results from CFD. The flame structure is shown to change dramatically across the bifurcations and a qualitative stability map is constructed.

\section{NUMERICAL FORMULATION}

The governing equations for turbulent combustion CFD are the compressible Navier-Stokes equations for mass, momentum, and energy conservation. A Reynolds-averaged approach is adopted and a second moment closure model is applied to calculate the Reynolds stresses [29]. A reaction progress variable is defined as

$$
c=\frac{Y_{F}-Y_{F R}}{Y_{F P}-Y_{F R}}
$$

where $Y_{F}$ denotes the mass fraction of fuel, and the subscripts $R$ and $P$ indicate the reactants and the products, respectively. The local flame properties such as temperature and density are determined from the reaction progress variable using the Bray-MossLibby (BML) flamelet relationships [30]. The transport equation for the Favre-mean reaction progress variable $\tilde{c}$ is

$$
\frac{\partial \bar{\rho} \tilde{c}}{\partial t}+\frac{\partial \bar{\rho} \tilde{u_{i}} \tilde{c}}{\partial x_{i}}=\frac{\partial}{\partial x_{i}}\left(\bar{\rho} \widetilde{u_{i}^{\prime \prime} c^{\prime \prime}}\right)+\overline{\dot{\omega}}
$$

The turbulent scalar flux $\widetilde{u_{i}^{\prime \prime} c^{\prime \prime}}$ is modelled using a standard gradient transport hypothesis [31]. The term $\overline{\dot{\omega}}$ is the mean turbulent reaction rate, expressed as the production rate of reaction progress variable per unit volume. This quantity is modelled using a laminar flamelet concept, in which combustion is assumed to take place within thin flame surfaces that separate the regions of reactants and products. Locally, turbulence acts to wrinkle and to distort these interfaces, however, the flame retains the local structure of a strained laminar flame.

The reaction rate source term is given by:

$$
\overline{\dot{\omega}}=\rho_{R} S_{L} I_{0} \Sigma
$$

The group of variables $\rho_{R} S_{L} I_{0}$ represents the reaction rate per unit flame area and $\Sigma$ is the surface area per unit volume. The density of the mixture is $\rho_{R}$, which is a known quantity. The unstrained laminar flame speed $S_{L}$ is obtained by means of an empirical correlation [18]. The correction factor $I_{0}$ accounts for the mean effects of strain and curvature on the laminar flamelet. This term is modelled from laminar flame computations with detailed chemistry [32] and from Direct Numerical Simulation (DNS) data from Bray and Cant [17], and was formulated by Brookes et al. [19] as:

$$
I_{0}=\int_{-\infty}^{a_{c}} P(a) d a-\mathrm{Ma} t_{r} \int_{0}^{a_{c}} a P(a) d a
$$

where $\mathrm{Ma}$ is the Markstein number, obtained from the experimental data of Searby and Quinard [33], $t_{r}$ is the laminar flame residence time and $a_{c}$ is the hydrodynamic strain rate at extinction. The probability density function $P(a)$ of the hydrodynamic strain rate is described by a Gaussian function with mean $\bar{a}$ and standard deviation $\sigma_{a}$ evaluated from DNS data [17].

The flame surface density (FSD) describes the effects of turbulence on the flame structure, and is quantified using an algebraic expression. The specific form of the model for the flame surface density was developed by Brookes et al. [19] and was calibrated against the stagnation-plate flame data of Cheng and Shepherd [34],

$$
\Sigma=\frac{C_{w} \bar{c}^{1.2}\left(1-\bar{c}^{0.8}\right)}{\hat{L}_{y}}
$$

The model constant $C_{w}=5.50 \times 10^{-3}$ and $\hat{L}_{y}$ is the integral length scale of flame wrinkling given by:

$$
\hat{L}_{y}=\frac{v}{S_{L}}\left(1+C_{f} \frac{u^{\prime}}{S_{L}}\right)^{-1}
$$


where $u^{\prime}$ is the turbulence intensity and the model constant $C_{f}$ is taken to be equal to 2.225 .

The model is implemented in the open source CFD toolkit OpenFOAM. The equations are discretized in space using a central differencing scheme which is second-order accurate in smooth regions of the solution and is flux limited to first-order near steep gradients in order to guarantee boundedness. An Euler-implicit time marching is employed to account for transient effects. The PISO pressure correction algorithm [35] is used to solve the system of equations. The algorithm splits the solution procedure into an implicit predictor step followed by two corrector steps. In the predictor step, all quantities except the pressure are updated by solving the momentum, energy and reaction progress variable equation, whilst in the corrector steps, the pressure Poisson equation is solved implicitly and other quantities are updated explicitly.

\section{DYNAMICAL SYSTEMS THEORY}

The state of a dynamical system is determined by its dynamical variables and evolves according to the equations of motion that govern the causal relation between the current and future states. In the present work, the bluff-body stabilized turbulent premixed flame is treated as a dynamical system. The change in the behaviour of the flame when a control parameter, for example the inlet velocity, is varied is known as a bifurcation. When the control parameter moves outside a linearly stable regime, the dynamical system transitions from a steady state without oscillation to a state with oscillation. The operating point at which the dynamical system transitions from a steady to an oscillating state is known as a Hopf point.

Phase space contains all possible dynamical states of a system. An attractor refers to a well-defined geometrical structure in the phase space that describes the asymptotic state of a nonlinear system. Each point contained in the phase space corresponds to a unique state of the dynamical system at a particular time. If there are $m$ dynamical variables, then the state at a given time can be represented by a point in the Euclidean space $\mathbb{R}^{m}$. The representative points trace out a path in the phase space, known as the phase trajectory. To construct the phase space, it is necessary to know the dimension of the phase space which is generally given by the number of the coupled set of ordinary differential equations (ODE). This quantity is often not known in advance and has to be estimated. The embedding theorem proposed by Takens [36] allows the reconstruction of a phase space from measurements obtained from experiments or CFD. This method asserts that if a time series is one component of an attractor that can be represented by a smooth $d$-dimensional manifold then the topological properties of the attractor are equivalent to the topological properties of the embedding formed by the $m$-dimensional phase space vectors.

$$
\begin{array}{r}
\vec{X}_{i}=(y(i \Delta t), y(i \Delta t+\tau), y(i \Delta t+2 \tau) \\
\ldots, y(i \Delta t+(m-1) \tau))
\end{array}
$$

where $i$ denote the hypersphere considered, $m$ is the embedding dimension and $\tau$ is the delay time. In this study the variable $y$ of interest is the pressure. The pressure is selected as we wish to understand how nonlinear hydrodynamics can affect the global flame behaviour, and ultimately how this would have an implication on thermoacoustic instability where pressure fluctuations are of primary importance. For a proper reconstruction, it is important to calculate the optimum delay time and the optimum embedding dimension. The minimum value of $m$ required to capture the topological properties of an attractor depends on the dimension of the attractor. The delay time has to be chosen carefully. This is because reconstructions with high correlations between consecutive phase space points will result if the delay times are too small, whereas the deterministic structure linking points along a single degree of freedom may be obscured for very large delays. Using the method proposed by Abarbanel [37], the optimal delay time is obtained by computing the first-zero crossing of the autocorrelation function.

The phase portrait is employed to visualize the reconstructed phase space. It is a three-dimensional plot of the system motion against that same motion shifted by one delay time and by two delay times. The topological manifold of the phase trajectory provides valuable information about the system dynamics. When the system is steady, a single point will be observed if no noise is present. For a limit cycle, the attractor is a closed curve, indicating that the solution is periodic in nature. For a quasiperiodic oscillation, two incommensurate frequencies are present, which modulate the amplitude of the solutions. As a result, the attractor will take the form of a torus in the phase space.

The topological dimension of an attractor is given by its correlation dimension. Like other fractal dimensions, it is used to identify the underlying dynamics of a system. By considering a simple Euclidean geometry, points have dimension 0, lines have dimension 1, plane surfaces have dimension 2, solids have dimension 3. Analogously in the examination of the dynamical system, the correlation dimension is zero for a steady flow (fixed point), one for a periodic limit cycle (closed trajectory) and two for quasiperiodicity (torus surface), fractional for chaotic motion (self-similar fractal object), and infinite for purely random noise.

\section{GEOMETRY AND BOUNDARY CONDITIONS}

The Volvo afterburner experiment consists of a rectangular duct of size $1.0 \mathrm{~m}$ (length) x $0.12 \mathrm{~m}$ (height) x $0.24 \mathrm{~m}$ (width) containing a triangular-shaped bluff body that extends between the two lateral walls in the enclosure and with its apex pointed 
towards the inlet. The sides of the bluff body triangular base measure $0.04 \mathrm{~m}$. The bluff-body is located $0.318 \mathrm{~m}$ downstream from the inlet of the duct. The problem is formulated using two dimensional planar coordinates by considering only the axial and transverse directions. The computational domain is divided into ten blocks as shown in Fig. 1. The mesh arrangement for the blocks are: blocks 1 and 2 consist of $280 \times 54$ mesh points; blocks 3 and 4 consist of $40 \times 54$ mesh points; blocks 5, 6 and 7 consist of $350 \times 54$ mesh points; blocks 8,9 and 10 consist of $350 \times 54$ mesh points. The mesh density around and downstream of the bluff-body (blocks 3-7) is increased so the overall flame structure at the stabilization point can be captured with sufficient resolution.

At the inlet, the axial velocity and temperature of the fully premixed propane-air mixture are fixed at $17 \mathrm{~m} / \mathrm{s}$ and $288 \mathrm{~K}$, respectively. A constant equivalence ratio of 0.65 for the fuel-air mixture is used. The progress variable is set to zero. The turbulence intensity and turbulence dissipation at the inlet are calculated based on the reported values from the experiment [39]. In the experiment [39], a low frequency pressure oscillation of around $100 \mathrm{~Hz}$ was reported. This provides an indication of the longitudinal acoustic mode in the combustor. To represent this, a wave-transmissive boundary condition is used at the outlet. The boundary condition is based on linear relaxation and the amplitude of the incoming characteristics is $\mathscr{L}=K\left(p-p_{\infty}\right)$, where $K$ is the stiffness parameter and $p_{\infty}$ is the far-field reference pressure [40]. The outlet becomes non-reflecting for small values of $K$ and becomes reflecting as $K$ increases. Here, the amplitude of the reflected wave is set to be close to one such that the boundary can be treated as partially reflecting.

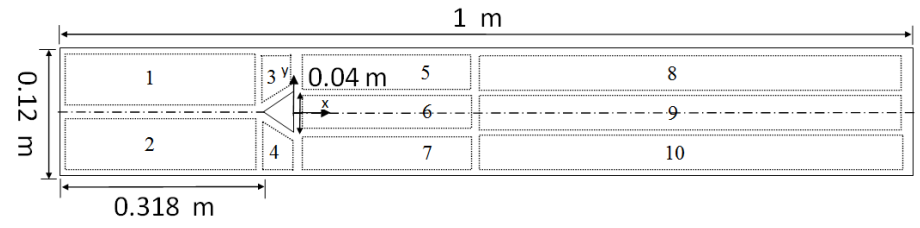

FIGURE 1: VOLVO EXPERIMENT (NOT TO SCALE)

The walls are treated as no-slip, impermeable and adiabatic. A zero gradient assumption is specified for the progress variable, temperature and pressure along the walls.

The computational domain is discretized such that resolution is sufficient to provide grid independence, since no discernible difference was observed in the flow field for both the cold and reacting flows after halving the number of mesh points in each direction. Once a steady non-reacting flow solution has been ob- tained, ignition is achieved by artificially fixing the value of the reaction progress variable at a particular position in the domain. The self-excitation occurs naturally in the calculation without imposing any external perturbations.

\section{RESULTS AND DISCUSSION}

Validation studies for the reacting flow are first performed using the data from the Volvo experiment [41,42] before considering the hydrodynamic behaviour of the flame. Both 2D and 3D URANS simulations are carried out to assess the accuracy of these two approaches [43]. These results are used to provide a preliminary evaluation in the ability of the URANS to represent the flow field and global flame structure. An instantaneous snapshot of the temperature field for the $2 \mathrm{D}$ reacting case is shown in Fig. 2(top) and the centreline time-averaged axial velocity is presented in Fig. 2(bottom). The flame is represented by a steep temperature gradient in Fig. 2(top), and can be visualized as sheetlike structures emanating from the upper and lower corners of the flameholder. The flame exhibits a symmetrical structure. The prediction of the recirculation zone length and the magnitude of the normalized axial velocity in the reacting flow is also in good agreement with experimental data (Fig. 2 bottom).
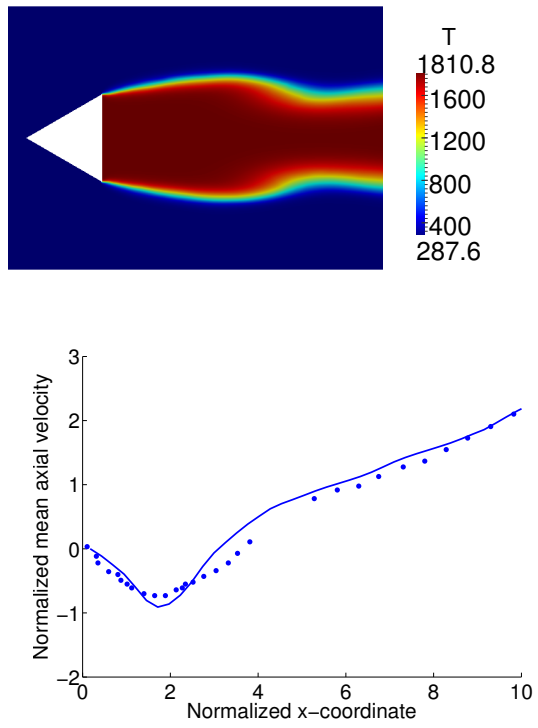

FIGURE 2: FLOW FEATURES FOR THE REACTING FLOW (TOP) INSTANTANEOUS TEMPERATURE FIELD (BOTTOM) CENTRELINE TIME-AVERAGED AXIAL VELOCITY PROFILES. POINTS (EXP) AND LINES (CFD)

Comparisons of the time-averaged axial and transverse ve- 
(a)

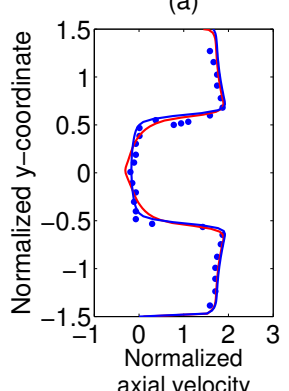

(f)

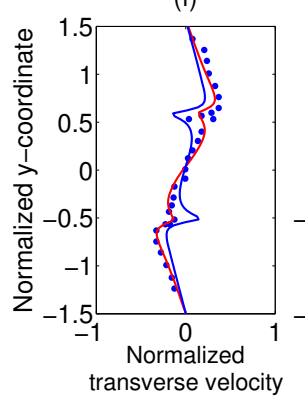

(b)

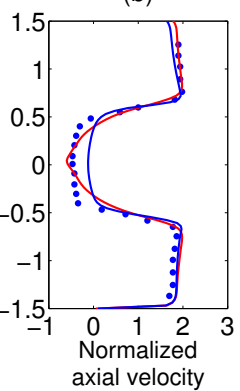

(g)

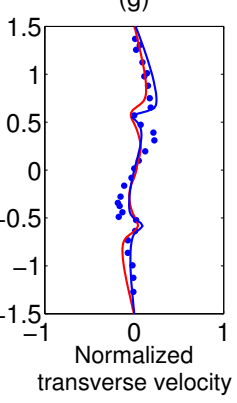

(c)

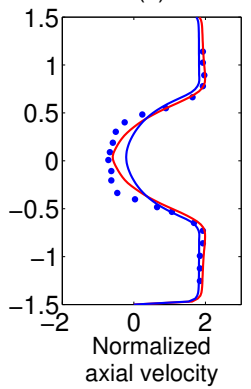

(h)

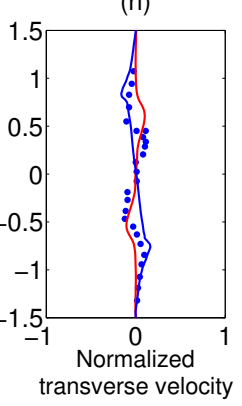

(d)
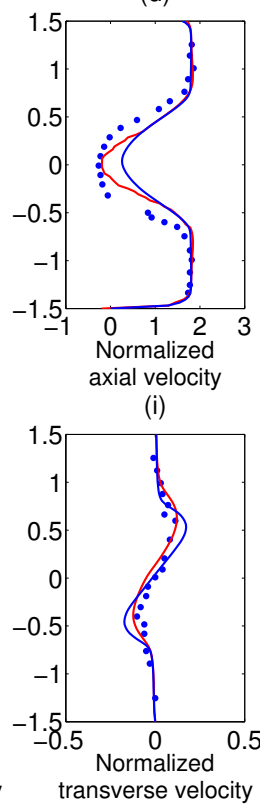

(e)

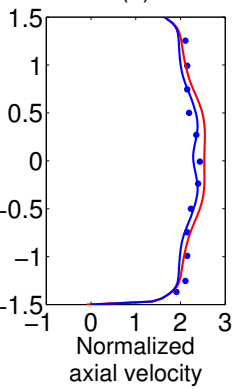

(j)

FIGURE 3: NORMALIZED AXIAL (a-e) AND TRANSVERSE VELOCITY (f-j) FOR THE REACTING FLOW AT $0.375 w_{f}, 0.95$ $w_{f}, 1.5 w_{f}, 3.75 w_{f}$ and $9.4 w_{f}$. POINTS: EXPERIMENT, BLUE LINES: RESULTS FOR 2D URANS AND RED LINES: RESULTS FOR 3D URANS

locity profiles for the reacting flow at five locations corresponding to $0.375 w_{f}, 0.95 w_{f}, 1.5 w_{f}, 3.75 w_{f}$ and $9.4 w_{f}$ are depicted in Fig. 3, where $w_{f}$ denotes the width of the flameholder. The time-averaged axial velocities are shown in the top row and the time-averaged transverse velocities are illustrated in the bottom row. Both the axial and transverse velocities are normalized against the inlet velocity. The points represent experimental data, the blue lines denote the numerical results for the 2D simulation and the red lines denote the numerical results for the 3D simulation. Good agreement between the numerical and experimental values is obtained. The normalized time-averaged axial velocity indicates that the recirculation zone remains prominent up to a downstream distance of $3.75 w_{f}$ (Fig. 3(d)). The axial velocity in the downstream region is almost two times larger than the inlet flow velocity due to the heat release in the wake, as shown in Fig. 3(e). Another notable aspect of the normalized transverse velocity is the inflection point in the shear layers around the flame stabilization region. This can be seen in the region of $y=0.5$ and $y=-0.5$ in Fig. 3(f) and (g). This behaviour corresponds to the rapid change in velocity across the flame front.

Results for the mean temperature profile at three locations $3.75 w_{f}, 8.75 w_{f}$ and $13.75 w_{f}$ are shown in Fig. 4. The temperature is normalized by the inlet temperature and the distance is normalized by the bluff body width. The normalized temperature field in the vicinity of the flameholder $3.75 w_{f}$ matches well

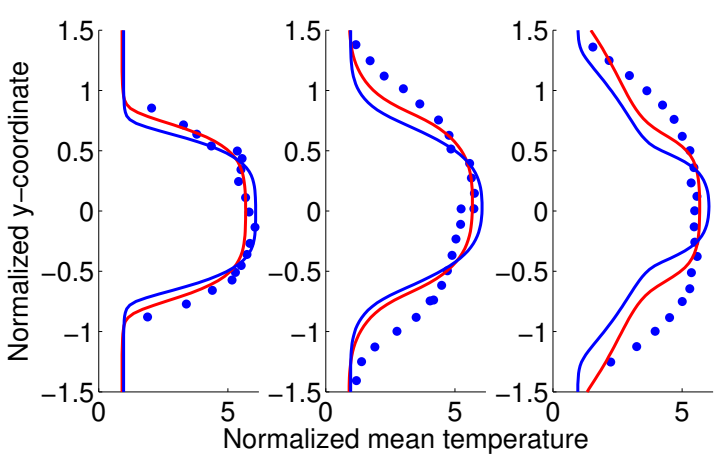

FIGURE 4: NORMALIZED TEMPERATURE PROFILE AT $3.75 w_{f}, 8.75 w_{f}$ and $13.75 w_{f}$. POINTS: EXPERIMENT, BLUE LINES: RESULTS FOR 2D URANS AND RED LINES: RESULTS FOR 3D URANS

with experimental data. Further downstream, underestimation of the spreading rate in the time-averaged temperature profile can be observed.

Results for the time-averaged velocities and temperature for the 3D case are denoted by the red lines in Fig. 3 and Fig. 4 respectively. In general, the 3D results offer a slight improvement 
in the agreement with experimental data for both velocities and temperature profiles at different axial locations. Visually, the 3D simulation results appear to be almost identical to the $2 \mathrm{D}$ simulation results extended in the spanwise direction. Despite the slightly better agreement, the computational cost for the unsteady 3D simulation using the RSM model is significantly higher and the improvement in the results does not justify its use for the present investigation. As such, the $2 \mathrm{D}$ simulation is used to investigate the effects of inlet velocity on the flame behaviour and extract the nonlinear dynamics on the system. It is recognized that the use of a 2D simulation implies that vortex breakdown is not properly accounted for. Nevertheless, for the cases of interest in this study, large-scale coherent motion perturbing the flame is expected to occur mainly along the longitudinal direction, and spanwise flow variation is assumed to be small in the mean. Small-scale flame wrinkling along the spanwise direction and in the far-field region is assumed not to have a significant effect on the global flame behaviour.

\section{EFFECTS OF INLET VELOCITY}

In this section, the inlet velocity is varied to investigate the nonlinear dynamics of the turbulent bluff-body flame. The Reynolds stresses are specified to provide a turbulence intensity of $4 \%$ of the mean inlet velocity for all cases investigated. All other parameters are kept constant. Six cases at different inlet velocity were simulated, as shown in Table 1.

\begin{tabular}{|cc|}
\hline Case & inlet velocity \\
\hline 1 & $14 \mathrm{~m} / \mathrm{s}$ \\
2 (validation) & $17 \mathrm{~m} / \mathrm{s}$ \\
3 & $21 \mathrm{~m} / \mathrm{s}$ \\
4 & $25 \mathrm{~m} / \mathrm{s}$ \\
5 & $29 \mathrm{~m} / \mathrm{s}$ \\
6 & $33 \mathrm{~m} / \mathrm{s}$ \\
\hline
\end{tabular}

\section{TABLE 1: CONDITIONS FOR NUMERICAL SIMULATION AT DIFFERENT INLET VELOCITY}

The normalized pressure (the amplitude of the pressure relative to its mean value and normalized by atmospheric pressure at $101325 \mathrm{~Pa}$ ) plotted against the inlet velocity is shown in Fig. 5. The pressures from the simulations are taken at three different locations, at $\mathrm{x}=0.325 \mathrm{~m}, 0.4 \mathrm{~m}$ and $0.5 \mathrm{~m}$ respectively downstream of the flameholder, and $y=0.038 \mathrm{~m}$ from the centreline. Fig. 5 shows that the amplitude of the normalized mean pressure varies with inlet velocity. The mean pressure is also observed to be invariant with spatial location at inlet velocities below $21 \mathrm{~m} / \mathrm{s}$. The increase in mean pressure is found to be small at the lower range of inlet velocities from $14 \mathrm{~m} / \mathrm{s}$ to $21 \mathrm{~m} / \mathrm{s}$. The amplitude of the mean pressure increases as the inlet velocity is increased from $21 \mathrm{~m} / \mathrm{s}$ to $25 \mathrm{~m} / \mathrm{s}$, undergoes a dip at $29 \mathrm{~m} / \mathrm{s}$ and increases once again at $33 \mathrm{~m} / \mathrm{s}$.

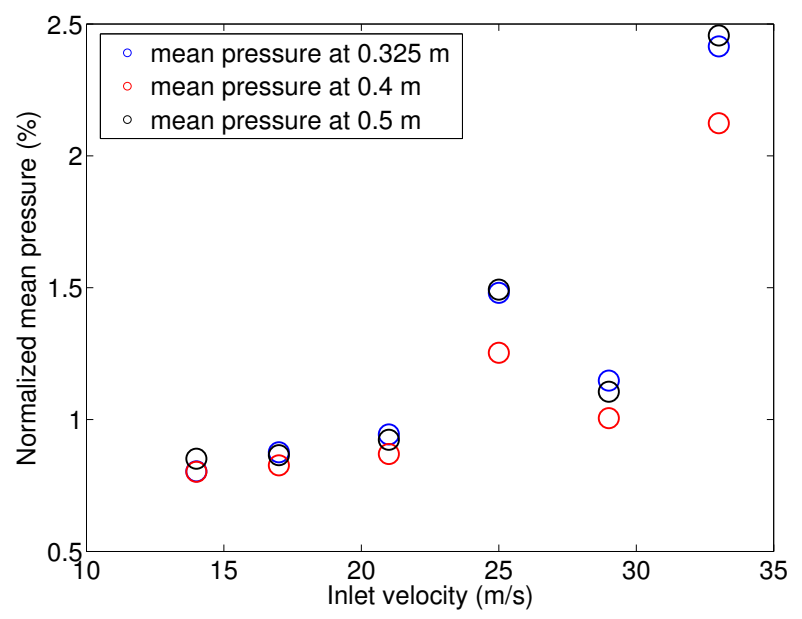

FIGURE 5: VARIATION OF MEAN PRESSURE (AS A PERCENTAGE OF THE ATMOSPHERIC PRESSURE) WITH INLET VELOCITY

The instantaneous behaviour of the flame is shown in Fig. 6 at each inlet velocity. The three columns in the figure correspond to reaction progress variable (first column), unsteady heat release (second column) and transverse velocity (third column). The flame structure exhibits distinct changes as the inlet velocity is increased. At $u_{\text {inlet }}=14 \mathrm{~m} / \mathrm{s}$ (first row), the flame is steady without much large-scale wrinkling. However, when the inlet velocity is increased to $u_{\text {inlet }}=17 \mathrm{~m} / \mathrm{s}$ (second row), some degree of unsteadiness is immediately evident. A similar flame behaviour is present at $u_{\text {inlet }}=21 \mathrm{~m} / \mathrm{s}$ (third row). At $u_{\text {inlet }}=25 \mathrm{~m} / \mathrm{s}$ (fourth row), the flame becomes highly unsteady and symmetrical rollup of the flame can be seen. An increase in the flame surface area is present as the flame front is perturbed strongly by the vortical structures formed due to the flow separation from the bluff body flameholder. The extent of rollup decreases significantly at $u_{\text {inlet }}$ $=29 \mathrm{~m} / \mathrm{s}$ (fifth row) and the flame reverts to a steady behaviour. At $u_{\text {inlet }}=33 \mathrm{~m} / \mathrm{s}$ (sixth row), the varicose flame structure as seen at $u_{\text {inlet }}=25 \mathrm{~m} / \mathrm{s}$ is absent. Instead, a sinuous flame structure can be seen. The out-of-phase behaviour between the upper and lower shear layer suggests that the strength of fluid-mechanical straining exceeds the baroclinic torque induced by the flame [1]. As a result, the flame is destabilized and strong hydrodynamic instabilities [13] become evident.

The magnitude of global heat release is found to increase 
(a)

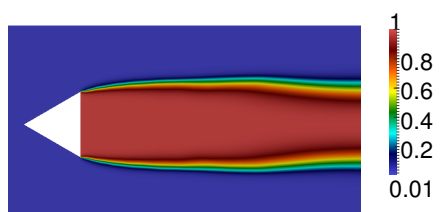

(b)

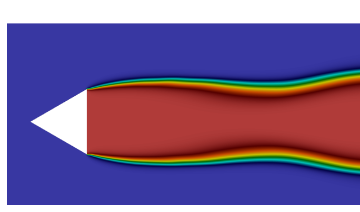

(c)

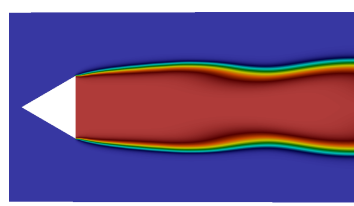

(d)

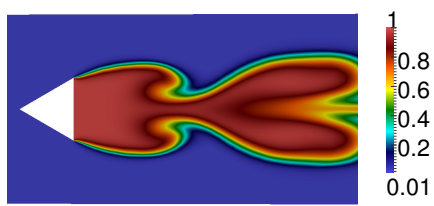

(e)

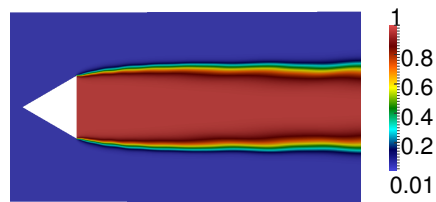

(f)

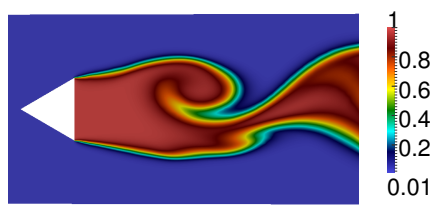

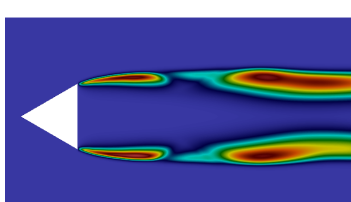
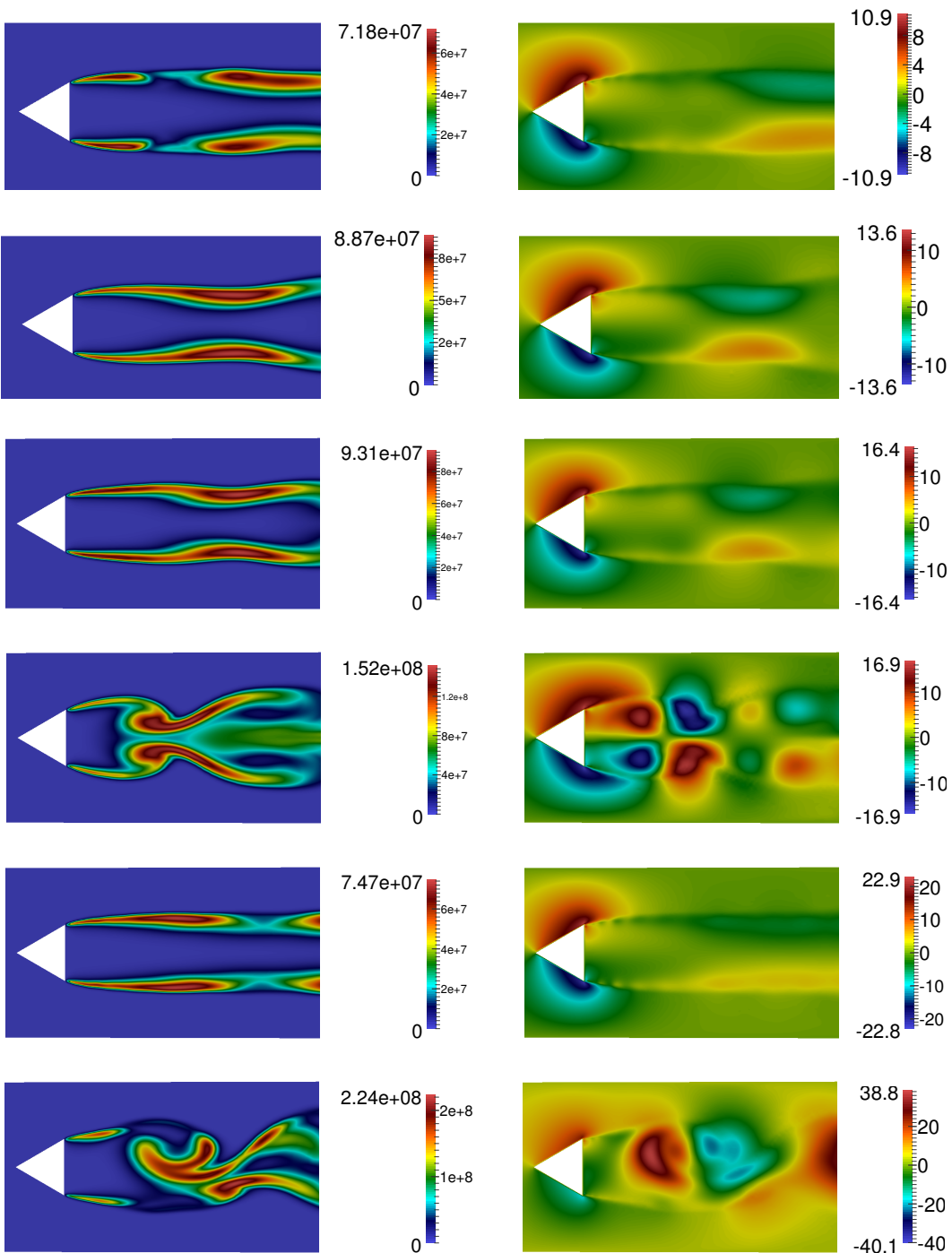

FIGURE 6: REACTION PROGRESS VARIABLE (LEFT), HEAT RELEASE RATE (MID) AND TRANSVERSE VELOCITY (RIGHT) AT $u_{\text {inlet }}$ (a) $14 \mathrm{~m} / \mathrm{s}$, (b) $17 \mathrm{~m} / \mathrm{s}$, (c) $21 \mathrm{~m} / \mathrm{s}$, (d) $25 \mathrm{~m} / \mathrm{s}$, (e) $29 \mathrm{~m} / \mathrm{s}$, (f) $33 \mathrm{~m} / \mathrm{s}$

with inlet velocity over the range considered, except at $u_{\text {inlet }}=$ $29 \mathrm{~m} / \mathrm{s}$. At $u_{\text {inlet }}=14 \mathrm{~m} / \mathrm{s}$ (first row), high heat release rate can be observed along the upper and lower separated shear layer of the recirculation zone. The magnitude of unsteady heat release increases as the flame undergoes high stretch close to the flow reversal region for $u_{\text {inlet }}=17 \mathrm{~m} / \mathrm{s}$ (second row) and $21 \mathrm{~m} / \mathrm{s}$ (third row). Large-scale coherent rollup of the symmetrical mode occurs at $u_{\text {inlet }}=25 \mathrm{~m} / \mathrm{s}$ (fourth row) is accompanied by an increase in heat release. A drop in the heat release, consistent with the reduction of large-scale unsteadiness, is evident at $u_{\text {inlet }}=29 \mathrm{~m} / \mathrm{s}$ (fifth row). Intense heat release can be seen as a result of the increase in flame surface area during the asymmetrical rollup at $u_{\text {inlet }}=33 \mathrm{~m} / \mathrm{s}$ (sixth row). Hydrodynamic effects on the flame structure are depicted by the contours of the transverse velocity. Small variations in the transverse velocity are present at $u_{\text {inlet }}=$ $14 \mathrm{~m} / \mathrm{s}$ when the flame is steady. Localized regions with higher transverse velocity can be seen at $u_{\text {inlet }}=17 \mathrm{~m} / \mathrm{s}$ and $21 \mathrm{~m} / \mathrm{s}$. At $u_{\text {inlet }}=25 \mathrm{~m} / \mathrm{s}$, regions with alternating signs of transverse velocity appear in the upper and lower halves of the duct along the shear layer indicating the presence of strong rollup. This behaviour disappears at $u_{\text {inlet }}=29 \mathrm{~m} / \mathrm{s}$. Instead, small-scale variations in transverse velocity are present along the separated shear layers behind the flameholder. This effect diminishes further downstream. The asymmetrical rollup of the the flame surface at $u_{\text {inlet }}=33 \mathrm{~m} / \mathrm{s}$ is shown by the alternate change in sign of the transverse velocity along the axial direction. 
(a)

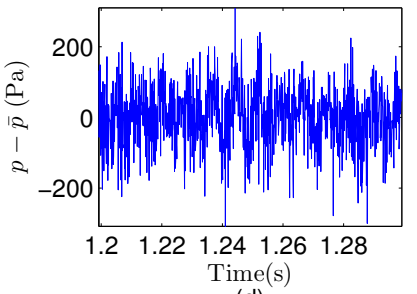

(d)

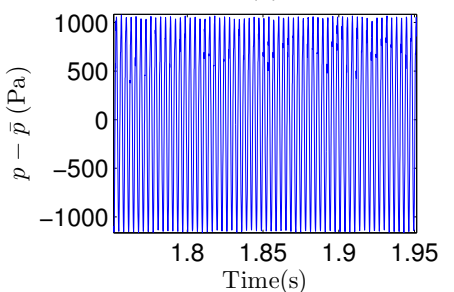

(b)

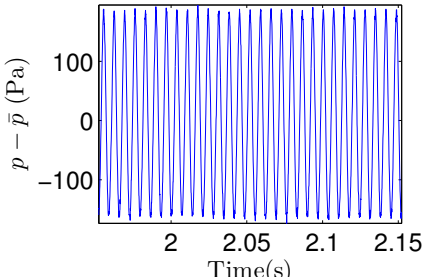

(e)

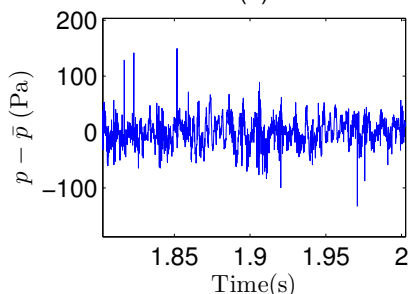

(c)

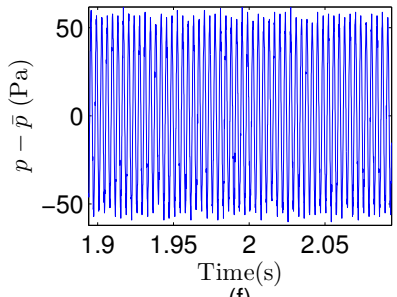

(f)

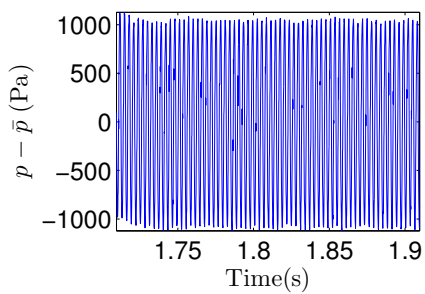

FIGURE 7: PRESSURE TIME SERIES AT DIFFERENT INLET VELOCITIES. (a) $u_{\text {inlet }}=14 \mathrm{~m} / \mathrm{s}$, (b) $u_{\text {inlet }}=17 \mathrm{~m} / \mathrm{s}$, (c) $u_{\text {inlet }}=21$ $\mathrm{m} / \mathrm{s}$, (d) $u_{\text {inlet }}=25 \mathrm{~m} / \mathrm{s}$, (e) $u_{\text {inlet }}=29 \mathrm{~m} / \mathrm{s}$, (f) $u_{\text {inlet }}=33 \mathrm{~m} / \mathrm{s}$

(a)

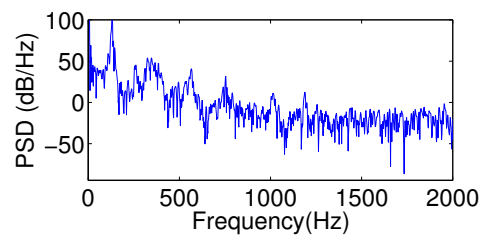

(b)

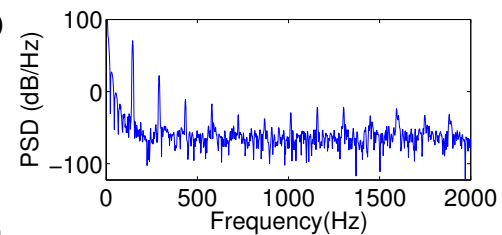

(c)

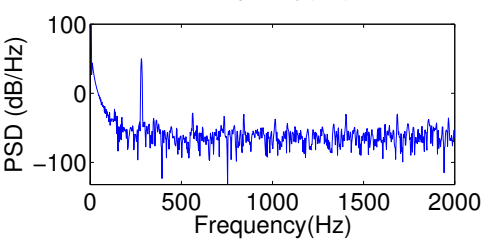

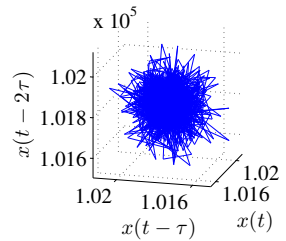
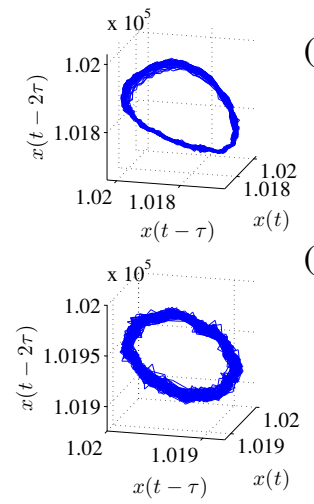

(d)

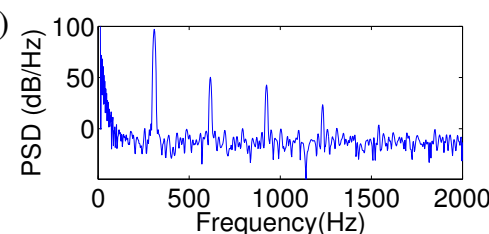

(e)

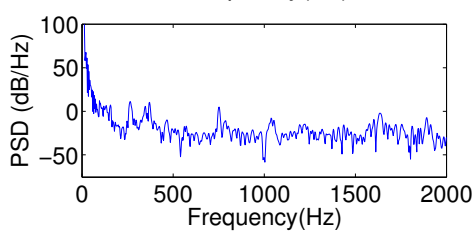

(f)

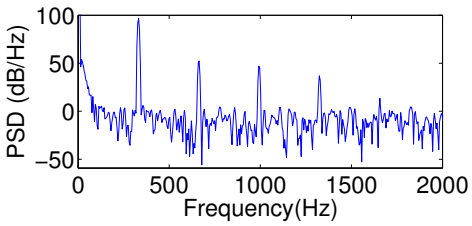

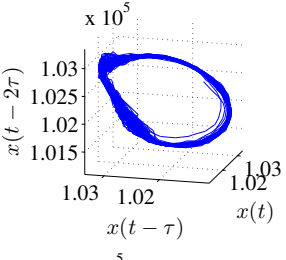
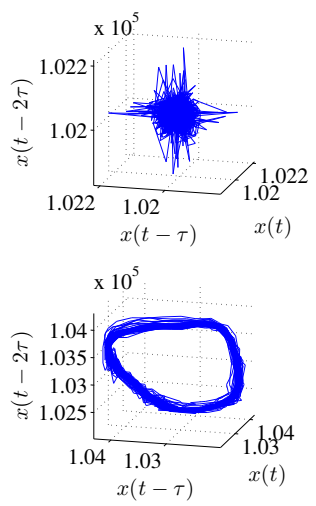

FIGURE 8: (LEFT) PSD; AND (RIGHT) PHASE PORTRAIT. a) $u_{\text {inlet }}=14 \mathrm{~m} / \mathrm{s}$, (b) $u_{\text {inlet }}=17 \mathrm{~m} / \mathrm{s}$, (c) $u_{\text {inlet }}=21 \mathrm{~m} / \mathrm{s}$, (d) $u_{\text {inlet }}=25$ $\mathrm{m} / \mathrm{s}$, (e) $u_{\text {inlet }}=29 \mathrm{~m} / \mathrm{s}$, (f) $u_{\text {inlet }}=33 \mathrm{~m} / \mathrm{s}$

The time series of the pressure fluctuations when the oscillation has saturated is shown in Fig. 7 to demonstrate the nonlinear behaviour of the dynamical system. At $u_{\text {inlet }}=14 \mathrm{~m} / \mathrm{s}$ (Fig. 7a), the pressure signal is incoherent as its amplitude fluctuates inconsistently with time. The pressure signal transitions to a sinusoidal waveform at $u_{\text {inlet }}=17 \mathrm{~m} / \mathrm{s}, 21 \mathrm{~m} / \mathrm{s}$ and $25 \mathrm{~m} / \mathrm{s}$ (Fig. $\left.7 \mathrm{~b}, \mathrm{c}, \mathrm{d}\right)$. The amplitude of the pressure fluctuations at $u_{\text {inlet }}=25 \mathrm{~m} / \mathrm{s}$ is however found to be much higher than at $u_{\text {inlet }}=17 \mathrm{~m} / \mathrm{s}$ and 21 $\mathrm{m} / \mathrm{s}$. This indicates the presence of a limit cycle oscillation due to strong organized vortical rollup. When the inlet velocity is increased to $u_{\text {inlet }}=29 \mathrm{~m} / \mathrm{s}$ (Fig. 7e), the pressure signal translates into a lower amplitude with less coherent behaviour. A possible cause for the sudden change in the dynamical behaviour is the transition of the flame from a varicose mode to a sinuous mode 
at $u_{\text {inlet }}=33 \mathrm{~m} / \mathrm{s}$ (Fig. 7f) or intermittent switching between the two modes. At $u_{\text {inlet }}=33 \mathrm{~m} / \mathrm{s}$, the pressure time series reverts back to its sinosoidal form with similar amplitude to that at $u_{\text {inlet }}$ $=25 \mathrm{~m} / \mathrm{s}$. The pressure signal is also found to be not perfectly sinusoidal, suggesting the presence of multiple harmonics of the fundamental frequency.

The frequency of oscillation can be revealed from the PSD of the pressure fluctuations shown in the left column of Fig. 8. The frequency of the oscillation increases with the inlet velocity. At $u_{\text {inlet }}=14 \mathrm{~m} / \mathrm{s}$ (Fig. 8a), the dominant frequency of flow oscillation is $107 \mathrm{~Hz}$. The Strouhal number is 0.31 based on the flameholder width of 0.04 and inlet velocity of $14 \mathrm{~m} / \mathrm{s}$. The PSD at $u_{\text {inlet }}=17 \mathrm{~m} / \mathrm{s}$ (Fig. 8b) shows a peak at $144 \mathrm{~Hz}$, which corresponds to a Strouhal number of 0.32 . At $21 \mathrm{~m} / \mathrm{s}$ (Fig. 8c), a dominant peak at $281 \mathrm{~Hz}$ (twice the frequency of $u_{\text {inlet }}=17 \mathrm{~m} / \mathrm{s}$ ) is present in the PSD, but its harmonics appear to be quite weak. A low amplitude peak at the first 'subharmonic' of the dominant frequency is also present in the PSD. Its presence suggests that the flame is undergoing a period-doubling motion, commonly associated with vortex pairing. At $u_{\text {inlet }}=25 \mathrm{~m} / \mathrm{s}$ (Fig. $8 \mathrm{~d}$ ), a spectral peak at the fundamental frequency of $312 \mathrm{~Hz}$, along with its harmonics, is evident in the PSD. The Strouhal number for this case is 0.49 . The presence of a distinct peak in the PSD is however not visible at $u_{\text {inlet }}=29 \mathrm{~m} / \mathrm{s}$ (Fig. 8e). A strong peak at $342 \mathrm{~Hz}$ corresponding to a Strouhal number of 0.42 appears once again at $u_{\text {inlet }}=33 \mathrm{~m} / \mathrm{s}$ (Fig. $8 \mathrm{f}$ ).

The phase portraits of the pressure fluctuations are plotted in the right column of Fig. 8 to illustrate the dynamics of the system. The trajectory of the phase portrait for the flame with $u_{\text {inlet }}=14 \mathrm{~m} / \mathrm{s}$ resembles a fixed point attractor. A distinct closed loop is evident in the phase portrait at $u_{\text {inlet }}=17-25 \mathrm{~m} / \mathrm{s}$. This demonstrates that the flame undergoes a limit cycle oscillation in this range of inlet velocities. It is also worth noting that the phase trajectory at $u_{\text {inlet }}=21 \mathrm{~m} / \mathrm{s}$ is slightly contaminated by background noise. A fixed point attractor affected by noise is revealed at $u_{\text {inlet }}=29 \mathrm{~m} / \mathrm{s}$. This is consistent with the appearance of the multiple peaks in the PSD which is a result of broadband excitation when the flame transitions from a varicose to sinuous mode. At $u_{\text {inlet }}=33 \mathrm{~m} / \mathrm{s}$, the system bifurcates into a limit cycle, confirming that a self-excited periodic oscillation takes place.

To verify the topological dimension of the attractor, the correlation sum $C$ and correlation dimension $D_{c}$ of the pressure signal are plotted in the left and right columns Fig. 9. The correlation dimension is computed using the Grassberger-Procaccia algorithm [38]. At $u_{\text {inlet }}=14 \mathrm{~m} / \mathrm{s}$ and $29 \mathrm{~m} / \mathrm{s}$, the correlation dimension $D_{c} \approx 0$ implies that the attractor is a fixed point. The correlation dimension $D_{c}$ cannot be obtained for the flame at both these inlet velocities because the pressure signal only contains random background noise over a mean value. Under this condition, $D_{c}=m$ because the number of resolvable degrees of freedom becomes limited by the reconstruction dimension. A limit cycle at $u_{\text {inlet }}=17-25 \mathrm{~m} / \mathrm{s}$ and $33 \mathrm{~m} / \mathrm{s}$ is indicated by $D_{c} \approx 1$ over a large range of Euclidean distance. At $u_{\text {inlet }}=17,25$ and 33 $\mathrm{m} / \mathrm{s}, D_{c} \approx 1$ over scales ranging from $R / R_{\max }$ from 0.05 to 0.5 and from $0.1 \leqslant R / R_{\max } \leqslant 0.5$ at $u_{\text {inlet }}=21 \mathrm{~m} / \mathrm{s}$.

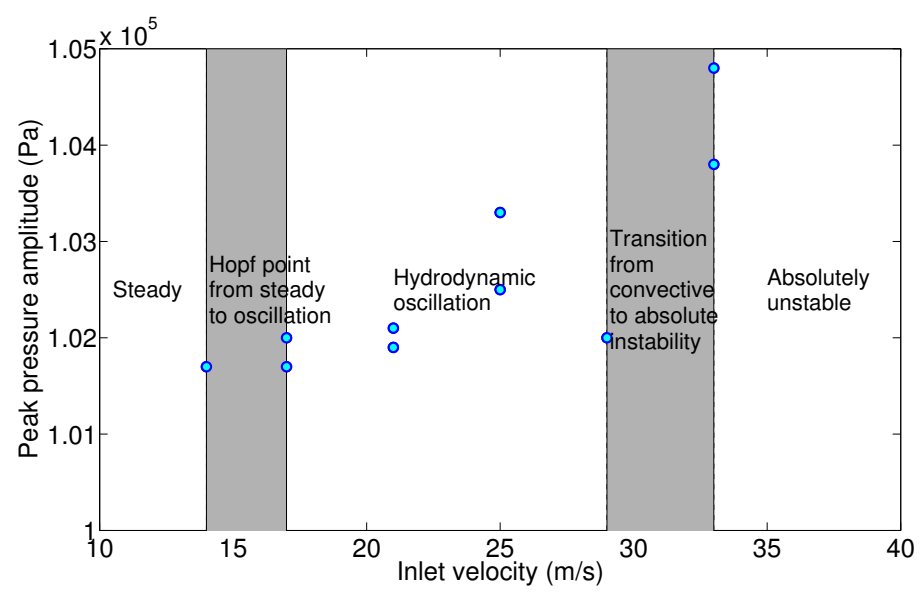

FIGURE 10: BIFURCATION DIAGRAM FOR INLET VELOCITY VARIATION

The global flame stability due to hydrodynamic effects can be understood by inspecting the bifurcation diagram which is shown in Fig. 10. The "stable" region is present when $u_{\text {inlet }}=$ $14 \mathrm{~m} / \mathrm{s}$ or lower, and is labelled as "steady". When the flame is steady, the pressure takes a single mean value and is affected only by random noise. Hydrodynamic oscillation has yet to be excited. Bifurcation of the flame to a limit cycle occurs at $u_{\text {inlet }}=$ $17 \mathrm{~m} / \mathrm{s}$. The same limit cycle behaviour is seen at $u_{\text {inlet }}=21 \mathrm{~m} / \mathrm{s}$. The transition from $u_{\text {inlet }}=17 \mathrm{~m} / \mathrm{s}$ to $u_{\text {inlet }}=21 \mathrm{~m} / \mathrm{s}$ occurs via a vortex merging process along with the presence of a 'subharmonic' frequency. The amplitude of the limit cycle increases at $u_{\text {inlet }}=25 \mathrm{~m} / \mathrm{s}$ where large-scale coherent structures of the symmetrical mode due to Kevin-Helmholtz instability are present. The limit cycle at $u_{\text {inlet }}=17,21$ and $25 \mathrm{~m} / \mathrm{s}$ are represented by two points in the bifurcation diagram and a distinct closed loop in the phase portrait. At $u_{\text {inlet }}=29 \mathrm{~m} / \mathrm{s}$, the flame reverts back to a "steady" state as it transitions from a symmetrical to an asymmetrical mode. It is important to note that this fixed point, however, is very sensitive to external noise and can bifurcate to a limit cycle very easily when subjected to a slight perturbation or with a change in the initial condition. The flame transitions back to a limit cycle once again at $u_{\text {inlet }}=33 \mathrm{~m} / \mathrm{s}$, but this time it exhibits a sinuous mode instead of the varicose behaviour observed at lower inlet velocities. The asymmetrical mode serves as an indication that the flow (and flame) is dominated by an absolutely unstable BVK instability $[1,44]$. 
(a)

(b)

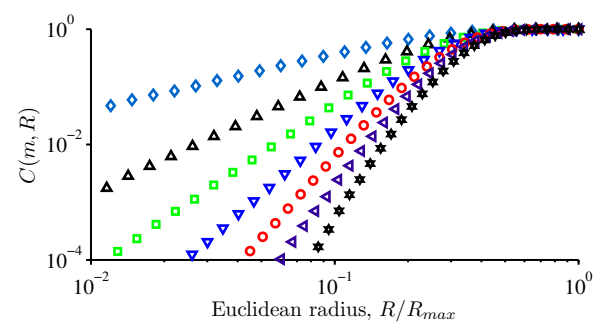

(c)

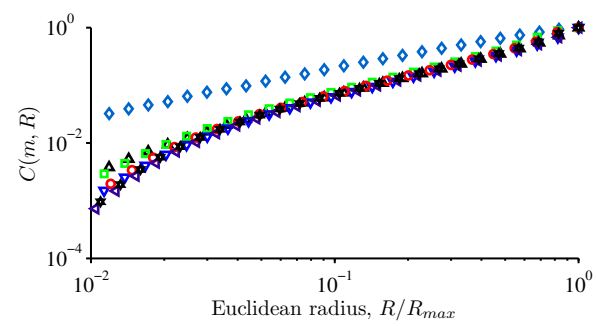

(d)
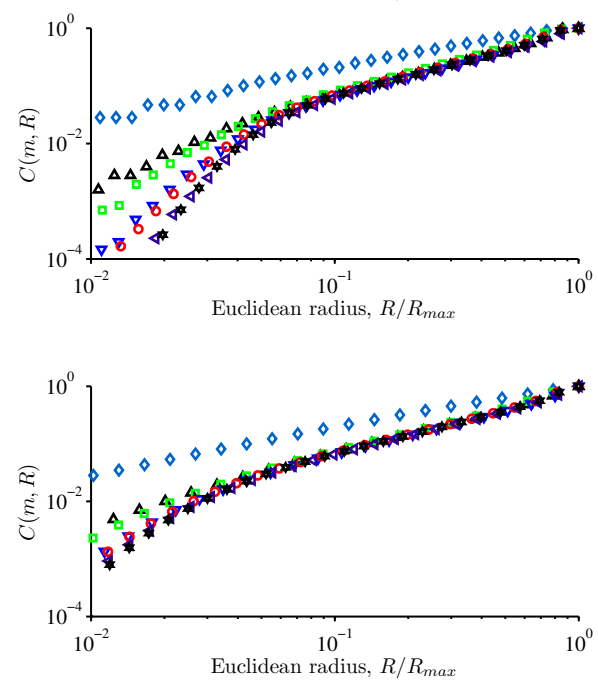

(f)
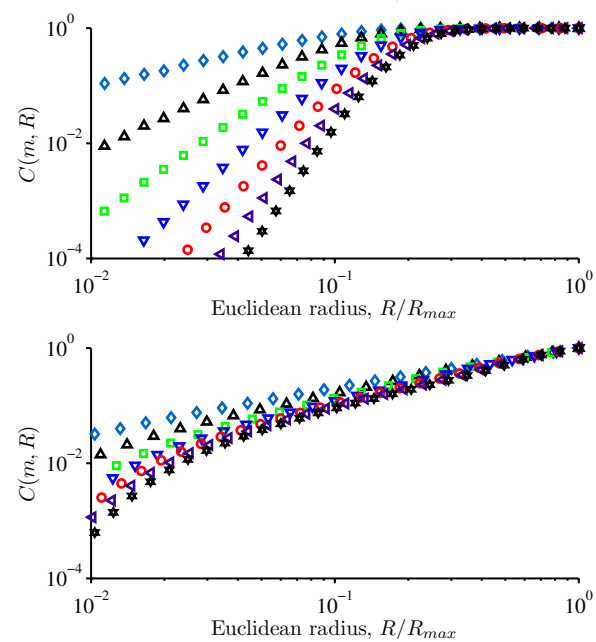

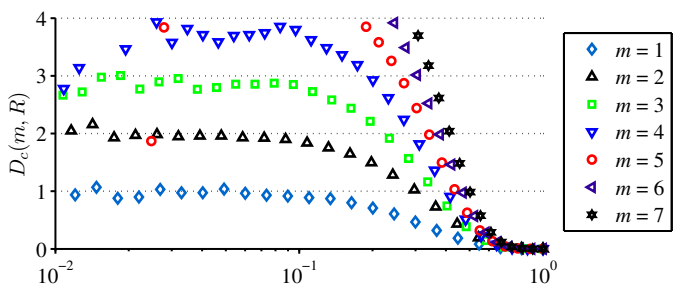

Euclidean radius, $R / R_{\max }$

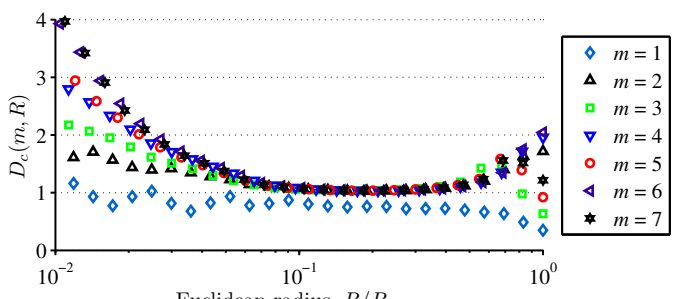

Euclidean radius, $R / R_{m a}$
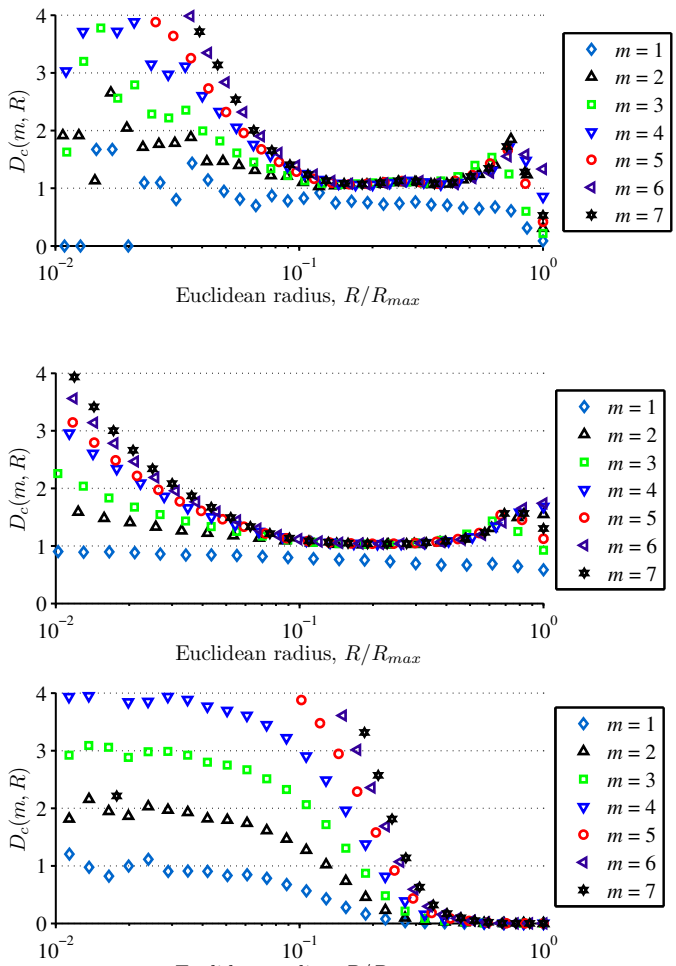

Euclidean radius, $R / R_{\max }$

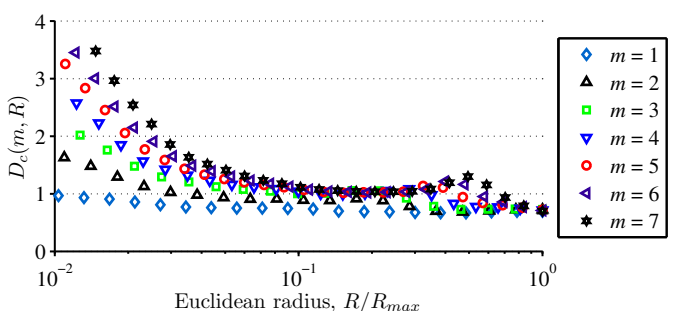

FIGURE 9: (LEFT) CORRELATION SUM AND (RIGHT) CORRELATION DIMENSION. a) $u_{\text {inlet }}=14 \mathrm{~m} / \mathrm{s},(\mathrm{b}) u_{\text {inlet }}=17 \mathrm{~m} / \mathrm{s},(\mathrm{c})$ $u_{\text {inlet }}=21 \mathrm{~m} / \mathrm{s}$, (d) $u_{\text {inlet }}=25 \mathrm{~m} / \mathrm{s}$, (e) $u_{\text {inlet }}=29 \mathrm{~m} / \mathrm{s}$, (f) $u_{\text {inlet }}=33 \mathrm{~m} / \mathrm{s}$ 


\section{CONCLUSION}

Nonlinear hydrodynamics of turbulent premixed bluff-body stabilized flames have been studied using turbulent combustion CFD with dynamical systems theory. The Volvo afterburner rig is used as the test case to elucidate the elaborate nonlinear dynamics exhibited by the flame, as well as changes in the global flame dynamics as the inlet velocity is varied. Validation cases for the reacting flow were first performed using 2D and 3D URANS. Results from the validation have shown that URANS is capable of representing the fundamental flow features and global flame structure adequately. Using dynamical systems theory, the flame was found to exhibit elaborate nonlinear behaviour with changes in inlet velocity. The flame is stable at $u_{\text {inlet }}=14 \mathrm{~m} / \mathrm{s}$, before transitioning into a limit cycle at $u_{\text {inlet }}=17 \mathrm{~m} / \mathrm{s}$. A limit cycle oscillation at $u_{\text {inlet }}=21 \mathrm{~m} / \mathrm{s}$ and $u_{\text {inlet }}=25 \mathrm{~m} / \mathrm{s}$ remains evident, except the amplitude of oscillation is much higher for the latter. The system then transitions back into a steady condition at $u_{\text {inlet }}=29 \mathrm{~m} / \mathrm{s}$ and undergoes a limit cycle oscillation again at $u_{\text {inlet }}=33 \mathrm{~m} / \mathrm{s}$. Unlike the symmetrical structure exhibited by the self-excited flame at lower velocities, the flame becomes strongly asymmetrical at $u_{\text {inlet }}=33 \mathrm{~m} / \mathrm{s}$. The presence of the sinuous mode suggests that the flame is absolutely unstable at $u_{\text {inlet }}$ $=33 \mathrm{~m} / \mathrm{s}$. The observed dynamical behaviour plays a crucial role in identifying and understanding the operating conditions where the flame is subjected to strong hydrodynamic instabilities. This approach can also serve as a first step in determining the key mechanisms that lead to the self-excited hydrodynamic oscillation, and therefore assist in developing methods to control flow instabilities.

\section{ACKNOWLEDGMENT}

The authors would like to express their appreciation for the financial support from the Dorothy Hodgkin Postgraduate Award and Rolls-Royce plc.

\section{REFERENCES}

[1] Shanbhogue, S. J., Husain, S., and Lieuwen, T., (2009). "Lean blowoff of bluff body stabilized flames: Scaling and dynamics". Progress in Energy and Combustion Science, 35, pp. 98-120.

[2] Dawson, J., Gordon, R., Kariuki, J., Mastorakos, E., Masri, A., and Juddoo, M., (2011). "Visualization of blow-off events in bluff-body stabilized turbulent premixed flames". Proceedings of the Combustion Institute, 33, pp. 15591566.

[3] Kariuki, J., Dawson, J. R., and Mastorakos, E., (2012). "Measurements in turbulent premixed bluff body flames close to blow-off". Combustion and Flame, 159, pp. 25892607.
[4] Schadow, K. C., Gutmark, E., Parr, T. P., Parr, D. M., Wilson, K. J., and Crump, J. E., (1989). "Large-scale coherent structures as drivers of combustion instability". Combustion Science and Technology, 64, pp. 167-186.

[5] Rolon, J. C., Candel, S., Renard, P.-H., and Thevenin, D., (2000). "Dynamics of flame/vortex interactions". Progress in Energy and Combustion Science, 26, pp. 225-282.

[6] Rayleigh, L., (1878). "The explanation of certain acoustical phenomena". Nature, pp. 319-320.

[7] O'Connor, J., Acharya, V., and Lieuwen, T., (2015). "Transverse combustion instabilities: Acoustic, fluid mechanic, and flame processes". Progress in Energy and Combustion Science, 49, pp. 1-39.

[8] Zukoski, E. E., and Marble, F. E., (1955). "The role of wake transition in the process of flame stabilization on bluff bodies". AGARD Combustion Researches and Reviews.

[9] Fujii, S., and Eguchi, K., (1981). "A comparison of cold and reacting flows around a bluff-body flame stabilizer". Journal of Fluids Engineering, 27, pp. 328-334.

[10] McMurtry, P. A., Riley, J. J., and Metcalfe, R. W., (1989). "Effects of heat release on the large-scale structure in turbulent mixing layers". Journal of Fluid Mechanics, 199, pp. 297-332.

[11] Fureby, C., and Löfström, C., (1994). "Large-eddy simulations of bluff body stabilized flames". Symposium (International) on Combustion, 25, pp. 1257-1264.

[12] Metcalfe, R. W., Orszag, S. A., Brachet, M. E., Menon, S., and Riley, J. J., (1987). "Secondary instability of a temporally growing mixing layer". Journal of Fluid Mechanics, 184, pp. 207-243.

[13] Erickson, R. R., and Soteriou, M. C., (2011). "The influence of reactant temperature on the dynamics of bluff body stabilized premixed flames". Combustion and Flame, 158, pp. 2441-2457.

[14] Briones, A., Sekar, B., Thornburg, H., and Granlund, K., (2011). "V-gutter stabilized turbulent premixed flame and lean blowout". International Symposium on Air Breathing Engines, September 12-16, Gothenborg, Sweeden, ISABE2011-1110.

[15] Poinsot, T., Trouve, A., Veynante, D., Candel, S., and Esposito, E., (1987). "Vortex-driven acoustically coupled combustion instabilities". Journal of Fluid Mechanics, 177, pp. 265-292.

[16] Rogers, D. E., and Marble, F. E., (1956). "A mechanism for high frequency oscillation in ramjet combustors and afterburners". Jet Propulsion, pp. 456-462.

[17] Bray, K. N. C., and Cant, R. S., (1991). "Some Applications of Kolmogorov's Turbulence Research in the Field of Combustion". Proceedings of the Royal Society A, 434, pp. 217-240.

[18] Abu-Orf, G. M., and Cant, R. S., (2000). “A turbulent reaction rate model for premixed turbulent combustion in spark- 
ignition engines". Combustion and Flame, 122, pp. 233252.

[19] Brookes, S. J., Cant, R. S., Dupere, I. D. J., and Dowling, A. P., (2001). "Computational modeling of self-excited combustion instabilities". Journal of Engineering for Gas Turbines and Power, 123, pp. 322-326.

[20] Zhu, M., Dowling, A. P., and Bray, K. N. C., (2001). "Selfexcited oscillations in combustors with spray atomizers". Journal of Engineering for Gas Turbines and Power, 123, pp. 779-786.

[21] Zhu, M., Dowling, A. P., and Bray, K. N. C., (2002). "Forced oscillations in combustors with spray atomizers". Journal of Engineering for Gas Turbines and Power, 124, pp. 20-30.

[22] Armitage, C. A., Balachandran, R., Mastorakos, E., and Cant, R. S., (2006). "Investigation of the nonlinear response of turbulent premixed flames to imposed inlet velocity oscillations". Combustion and Flame, 146, pp. 419-436.

[23] Dowling, A. P., (1997). "Nonlinear self-oscillation of a ducted flame". Journal of Fluid Mechanics, 346, pp. 271290.

[24] Noiray, N., Durox, D., Schuller, T., and Candel, S. M., (2008). "A unified framework for nonlinear combustion instability analysis based on the flame describing function". Journal of Fluid Mechanics, 615, pp. 139-167.

[25] Durox, D., Schuller, T., Noiray, N., and Candel, S., (2009). "Experimental analysis of nonlinear flame transfer functions for different flame geometries". Proceedings of the Combustion Institute, 32, pp. 1391-1398.

[26] Kashinath, K., Hemchandra, S., and Juniper, M. P., (2013). "Nonlinear thermoacoustics of ducted premixed flames: The influence of perturbation convection speed". Combustion and Flame, 160, pp. 2856-2865.

[27] Kashinath, K., Waugh, I. C., and Juniper, M. P., (2014). "Nonlinear self-excited thermoacoustic oscillations of a ducted premixed flame: bifurcations and routes to chaos". Journal of Fluid Mechanics, 761, pp. 399-430.

[28] Kabiraj, L., Wahi, P., and Sujith, R., (2011). "Bifurcations of self-excited ducted laminar premixed flames". Journal of Engineering for Gas Turbines and Power, 134, pp. 1-7.

[29] Launder, B. E., Reece, G. J., and Rodi, W., (1975). "Progress in the development of a Reynolds-stress turbulence closure". Journal of Fluid Mechanics, 68, pp. 537566.

[30] Bray, K. N. C., Libby, P. A., and Moss, J. B., (1985). "Unified modeling approach for premixed turbulent combustion - Part I : general formulation". Combustion and Flame, 61, pp. 87-102.

[31] Daly, B. J., and Francis, H. H., (1970). "Transport equations in turbulence". Physics of Fluids, 13, pp. 2634-2649.

[32] Cant, R. S., Bray, K. N. C., Kostiuk, L. W., and Rogg, B., (1994). "Flow divergence effects in strained laminar flamelets for premixed turbulent combustion". Combustion Science and Technology, 95, pp. 261-276.

[33] Searby, G., and Quinard, J., (1990). "Direct and indirect measurements of Markstein numbers of premixed flames". Combustion and Flame, 82, pp. 298-311.

[34] Cheng, R. K., and Shepherd, I. G., (1991). “The Influence of Burner Geometry on Premixed Turbulent Flame Propagation". Combustion and Flame, 26, pp. 7-26.

[35] Issa, R. I., (1985). "Solution of the implicitly discretised fluid flow equations by operator-splitting”. Journal of Computational Physics, 62, pp. 40-65.

[36] Ruelle, D., and Takens, F., (1971). "On the nature of turbulence". Communications in Mathematical Physics, 20, pp. 303-322.

[37] Abarbanel, H. D. I., (1996). "Analysis of Chaotic Data". Springer-Verlag, New York, NY USA.

[38] Grassberger, P., and Procaccia, I., (1983). "Characterization of strange attractors". Physical Review Letter, 50, pp. 346349.

[39] Sjunnesson, A., Henrikson, P., and Lofstrom, C., (1992). "CARS measurement and visualization of reacting flow in a bluff body stabilized flame". AIAA/SAE/ASME/ASEE 28th Joint Propulsion Conference and Exhibit, July 6-8, Nashville, TN.

[40] Poinsot, T., and Lele, S., (1992). "Boundary conditions for direct simulations of compressible viscous flows". Journal of Computational Physics, 101, pp. 104-129.

[41] Sjunnesson, A., Olovsson, S., and Sjöblom, S., (1991). "Validation rig - A tool for flame studies". In ISABE Conference, Nottingham, UK.

[42] Sjunnesson, A., Olovsson, A., and Max, E., (1991). "Measurements of velocities and turbulence in a bluff body stabilized flame". In Fourth International Conference on Laser Anemometry - Advances and Application, ASME Cleveland, US.

[43] Lee, C. Y., (2015). "Numerical investigation of combustion instabilities in afterburners". PhD Thesis, University of Cambridge.

[44] Prasad, A., and Williamson, C. H. K., (1997). "The instability of the shear layer separating from a bluff body". Journal of Fluid Mechanics, 333, pp. 375-402. 